\title{
The Current Status of Indoor Garden Design and Construction in Service Areas on Korea's Expressways
}

\author{
Kyoung-Ok Choi* and Jong-Eun Song \\ Department of Horticulture, Gyeongnam National University of Science and Technology, Jinju 52725, South Korea
}

\begin{abstract}
The purpose of this study is to investigate the current status of the construction of indoor gardens in expressway service areas. This study can be utilized as basic data for efficient and successful indoor garden introduction in future service areas and improvements for current service areas on expressways. The study period was from September 2, 2016 to October 11, 2017. The status of indoor garden was determined by visiting 147 expressway service areas in Korea. Physical environment survey items include architectural structure, usage type, facility and garden ornament, mobility, material of planter, soil type and planting plants. Among the 147 major expressway service areas, 56 were constructed with indoor gardens done, showing an indoor garden construction ratio of $38 \%$. In terms of the number of service areas with indoor gardens in expressway, Gyeongbu Expressway had the largest number of 16 areas. This was followed by 10 in Jungang Highway, 8 in the Jungbunaeryuk Expressway, 6 in the Seohaean Expressway, 4 in the Namhae Expressway, 4 in the Youngdong Expressway, 3 in the Donghaean Expressway, 3 in Tongyeong-daejeon Jungbu Expressway, and 2 in Honam Expressway. Thirty five service areas in expressway were constructed with natural flowers, 7 service areas were decorated with artificial flowers, and 14 service areas were arranged with both natural and artificial flowers. Most of the indoor gardens in the expressway were constructed in the restroom space, 47 in the men's restroom and 50 in the women's restroom. The indoor garden landscape in the expressway service area was all designed as a fixed garden type, except for three places composed of portable planters. There were 19 families, 27 genera, and 30 species of plants found in the indoor gardens in the expressway service areas.
\end{abstract}

Keywords: interior landscape, interior plants, service areas planting concept

\section{Introduction}

Gyeongbu Expressway, which has the Route number 1 in South Korea, was opened on December 23, 1968 from Seoul to Suwon (23.6 km) and completed in July 1970 connecting to Busan, and was officially opened in August 1971. Korea's first expressway service area is Chupungryeong Service Area (Lee et al., 2015), and as Naerincheon and Hongcheon Service Area also opened along with Seoul-Yangyang Expressway in June 2017, there are currently 189 expressway service areas in Korea (Korea Expressway Corporation, 2017).

An expressway service area is the hub of traffic and transportation that reflects the region's image, and is the central

This study was supported by 2016 School Supporting Association of Gyoungnam National University of Science and Technology.

Received: January 29, 2018, Revised: February 9, 2018, Accepted: February 11, 2018

First author: Kyoung-Ok Choi, E-mail: doctorcko@gmail.com, ORCID: 0000-0001-7990-7606

*Corresponding author: Kyoung-Ok Choi, E-mail: doctorcko@gmail.com, ORCID: 0000-0001-7990-7606 
space where many people come and go. However, while approximately 3.2 million vehicles a day are stopping by expressway service areas, they are merely serving as amenities or facilities for convenience such as to take a break, use the restrooms, refuel the car, or grab a bite (Choi and Lee, 2014). Most expressway service areas lack the ability to provide a pleasant resting place or efficient eco-friendly space for users.

According to studies on green space at expressway service areas in Korea, landscape space in service areas does not show differentiated features among service areas due to standardized establishment and implementation of plans according to the expressway landscaping standard drawing (Lee and Kim, 2011). In addition, landscape spaces and green areas have been eaten up rapidly due to the expansion of utilities and excessive implementation of commercial facilities led by increased users of expressway service areas (Lee et al., 2015). Moreover, Lee and Kim (2011) stated that it is necessary to create diverse and stereoscopic landscapes, increase the revegetation ratio, and expand resting places with nature in terms of improving design components of green spaces at the Youngdong Expressway service area. However, there has been almost no research on the indoor garden design in expressway service areas in Korea thus far.

Recently, garden-type interior landscapes have been widely constructed in expressway service areas as part of the policy to improve the environment and create a pleasant and beautiful expressway service area. Therefore, this study is conducted to examine the current state of indoor garden design in expressway service areas and determine the components of plants, garden ornaments and landscaping, thereby seeking effective ways to implement interior landscape.

\section{Research Method}

This study was conducted from September 2, 2016 to October 11, 2017. The current state of interior landscaping was determined by visiting 147 expressway service areas of 9 routes, such as Gyeongbu, Namhae, Donghaean, Seohaean, Youngdong, Jungbunaeryuk, Jungang, Tongyeong-Daejeon Jungbu and Honam Expressway, out of 189 expressway service areas nationwide operated by Korea Expressway Corporation.

The location of interior landscape in service areas was first examined, followed by cultivated plants, decorations of artificial flowers, and decorations of both plans and artificial flowers. The installation type, usage type mobility, and planter material were surveyed in both men's and women's restrooms where interior landscape is installed. For the installation type, we distinguished the special combination type from general landscape type, such as garden type, planter-box type, artificial flower type, fountain type, and aquarium (Yun at al., 2016), We categorized usage type into appreciation, experience, and rest according to how users use interior landscape (Shin et al., 2013), and for mobility we determined whether the landscape is fixed type or portable type. We classified planter material into marble, glass and preservative wood.

Items such as garden ornaments or soil of interior landscape were also surveyed. The planting type of plants in the interior landscape is classified into upper, middle and lower plants. This study is limited in that it examined the current state of interior landscape in expressways nationwide, thereby not conducting statistical analyses aside from frequency analysis.

\section{Results and Discussions}

\section{Current state of interior landscaping in expressway service areas}

There were total 147 service areas in 9 major expressway routes in Korea, and 56 of them had interior landscaping, showing the ratio of $38.0 \%$ overall (Table 1). As for the number and ratio of service areas with interior landscaping per 
Table 1. The installation status of indoor garden in expressway service areas.

\begin{tabular}{lcc}
\hline \multicolumn{1}{c}{ Expressway routes } & No. of Service areas & No. of Indoor garden (\%) \\
\hline Gyeongbu & 34 & $16(47.0)$ \\
Namhae & 13 & $4(30.7)$ \\
Donghaean & 6 & $3(50.0)$ \\
Seohaean & 18 & $6(33.3)$ \\
Youngdong & 13 & $4(30.7)$ \\
Jungbunaeryuk & 16 & $8(50.0)$ \\
Jungang & 14 & $10(71.4)$ \\
Tongyeong-daejeon Jungbu & 18 & $3(16.6)$ \\
Honam & 15 & 147 \\
Total & & $2(13.3)$ \\
\hline
\end{tabular}

expressway route, there were 16 on Gyeongbu Expressway (47\%), 4 on Namhae Expressway (30.7\%), 3 on Donghaean Expressway (50\%), 3 on Seohaean Expressway (33.3\%), 4 on Youngdong Expressway (30.7\%), 8 on Jungbunaeryuk Expressway (50\%), 10 on Jungang Expressway (71.4\%), 3 on Tongyeong-Daejeon·Jungbu Expressway (16.6\%), and 2 on Honam Expressway (13.3\%). Compared to Gyeongbu Expressway, only 2 service areas on Honam Expressway had interior landscape installed, showing a remarkable difference. This low installation ratio of interior landscape is due to the fact that expressway service areas in Korea are still mostly for satisfying the physiological needs for long-distance travel, refueling, or selling commercial goods such as foods and beverages (Kwon et al., 2010), while lacking perception about improving resting places or serving as an interior landscape (Lee et al., 2015). Accordingly, it seems necessary to implement efficient interior landscaping for visitors at expressway service areas to feel refreshed, relieve fatigue, and enjoy a pleasant and beautiful atmosphere.

\section{Location of indoor garden in service areas and current state of decoration type}

The expressway interior landscapes were mostly in the restrooms (Table 2), with 47 gardens in men's restrooms and 50

Table 2. Number of indoor gardens by location in expressway service areas.

\begin{tabular}{lccc}
\hline \multicolumn{1}{c}{ Expressway routes } & Men's restroom & Women's restroom & Other places \\
\hline Gyeongbu & 12 & 13 & 4 \\
Namhae & 4 & 3 & 2 \\
Donghaean & 2 & 3 & 1 \\
Seohaean & 5 & 6 & 4 \\
Youngdong & 4 & 4 & 2 \\
Jungbunaeryuk & 8 & 8 & 1 \\
Jungang & 7 & 9 & 5 \\
Tongyeong-daejeon Jungbu & 3 & 2 & 2 \\
Honam & 2 & 2 & 50 \\
Total & 47 & & 2 \\
\hline
\end{tabular}


Table 3. Number of indoor gardens in each decoration type in expressway service areas.

\begin{tabular}{|c|c|c|c|}
\hline Expressway routes & $\begin{array}{l}\text { Decoration with } \\
\text { natural flowers }\end{array}$ & $\begin{array}{l}\text { Decoration with } \\
\text { artificial flowers }\end{array}$ & $\begin{array}{l}\text { Decoration with natural } \\
\& \text { artificial flower }\end{array}$ \\
\hline Gyeongbu & 10 & 2 & 4 \\
\hline Namhae & 2 & 2 & \\
\hline Donghaean & 1 & & 2 \\
\hline Seohaean & 6 & & \\
\hline Youngdong & 2 & 1 & 2 \\
\hline Jungbunaeryuk & 3 & & \\
\hline Jungang & 5 & 1 & 5 \\
\hline Tongyeong-daejeon Jungbu & 4 & 1 & 1 \\
\hline Honam & 2 & & \\
\hline Total & 35 & 7 & 14 \\
\hline
\end{tabular}

in women's restrooms, indicating that there were slightly more interior landscapes in women's restrooms.

There were 35 expressway service areas decorated with natural flowers, 7 decorated with artificial flowers, and 14 decorated with a combination of both natural and artificial flowers (Table 3 ).

\section{Indoor garden installation type, usage type and planter material in expressway service areas}

This study analyzed the installation type, usage type, mobility and planter material of interior landscape installed in men's and women's service areas for each expressway route of Gyeongbu, Namhae, Donghaean, Seohaean, Youngdong, Jungbunaeryuk, Jungang, Tongyeong-Daejeon Jungbu, and Honam Expressway (Table 4-10). As a result of examining the installation type of interior landscapes, 81 of them were general garden type with mixed plantations of multiple plants, taking up most of the landscapes, 4 were artificial flower type with a combination of special forms aside from plants, 4 were planter-box type, 1 was fountain type, and 3 were aquarium type (Yun at al., 2016). For usage type, all landscapes were to appreciate the indoor gardens without installing facilities for experience or rest (Shin et al., 2013). The mobility survey showed that all gardens were fixed type except for 3 places (Table 4-10).

For the types of interior landscapes installed in men's and women's restrooms at Gyeongbu Expressway service areas, 18 were garden type, followed by 3 planter-box type and 1 fountain type. They were all for appreciation and installed as fixed type except for 1 portable type. Marble was most commonly used planter material, followed by installation of both marble and preservative wood, and then glass. Some service areas had interior landscape only on either men's or women's restrooms (Table 4). Namhae Expressway and Donghae Expressway service areas also had the landscapes installed in garden type for appreciation in fixed form, with marble and glass used as most common planter materials (Table 5, 6). Haengdamdo Service Area on Seohaean Expressway had a single-file parallel type interior landscape, most for appreciation and in fixed type (Table 7).

The interior landscape installed in Chilseo Service Area on Jungbunaeryuk Expressway was aquarium type, while others were garden type installed for appreciation in fixed form, using marble and glass (Table 9). At Andong Service Area on Jungang Expressway, women's restroom had the interior landscape installed in planter-box type, while other service areas were garden type installed for appreciation in fixed form, using marble (Table 10). In Deogyusan Service 
Table 4. Installation and usage type of indoor garden in Gyeongbu expressway service areas.

\begin{tabular}{|c|c|c|c|c|c|}
\hline Name of service & & Installation type & Usage type & Mobility & Material of planter \\
\hline \multirow{2}{*}{ Geoncheon } & $\mathrm{M}^{\mathrm{z}}$ & Garden type & Appreciation & Fixed type & Marble, Preservative wood \\
\hline & W & Garden type & Appreciation & Fixed type & Marble, Preservative wood \\
\hline \multirow{2}{*}{ Gyeongsan } & $\mathrm{M}$ & Garden type & Appreciation & Fixed type & Marble \\
\hline & W & Garden type & Appreciation & Fixed type & Glass \\
\hline \multirow{2}{*}{ Gyeongju } & M & Artificial flower type & Appreciation & Fixed type & Preservative wood \\
\hline & W & - & - & - & - \\
\hline \multirow{2}{*}{ Geumgang } & M & Planter-box type & Appreciation & Fixed type & \\
\hline & W & Garden type & Appreciation & Fixed type & Preservative wood \\
\hline \multirow{2}{*}{ Gimcheon } & M & Garden type & Appreciation & Fixed type & Glass \\
\hline & $\mathrm{W}$ & Garden type & Appreciation & Fixed type & Glass \\
\hline \multirow{2}{*}{ Manghyang } & $\mathrm{M}$ & Garden type & Appreciation & Fixed type & Marble \\
\hline & W & Garden type & Appreciation & Fixed type & Marble, Preservative wood \\
\hline \multirow{2}{*}{ Sintanjin } & $\mathrm{M}$ & Artificial flower type & Appreciation & Fixed type & Marble \\
\hline & W & Garden type & Appreciation & Fixed type & Marble \\
\hline \multirow{2}{*}{ Anseong } & M & - & - & - & - \\
\hline & $\mathrm{W}$ & Planter-box type & Appreciation & Portable type & Marble \\
\hline \multirow{2}{*}{ Okcheon } & M & Garden type & Appreciation & Fixed type & Marble, Preservative wood \\
\hline & W & Garden type & Appreciation & Fixed type & Marble, Preservative wood \\
\hline \multirow{2}{*}{ Ipjang } & $\mathrm{M}$ & - & - & - & - \\
\hline & $\mathrm{W}$ & Fountain type & Appreciation & Fixed type & Marble \\
\hline \multirow{2}{*}{ Jugam } & M & - & - & - & - \\
\hline & W & Garden type & Appreciation & Fixed type & Marble \\
\hline \multirow{2}{*}{ Cheonan } & $\mathrm{M}$ & Planter-box type & Appreciation & Portable type & \\
\hline & W & - & - & - & - \\
\hline \multirow{2}{*}{$\begin{array}{l}\text { Cheonan } \\
\text { (Samgeori direction) }\end{array}$} & M & - & - & - & - \\
\hline & $\mathrm{W}$ & Aquarium type & Appreciation & Fixed type & Glass, Marble \\
\hline \multirow{2}{*}{$\begin{array}{l}\text { Cheongju } \\
\text { (Cheongwon direction) }\end{array}$} & M & Garden type & Appreciation & Fixed type & Marble \\
\hline & $\mathrm{W}$ & Garden type & Appreciation & Fixed type & Preservative wood \\
\hline \multirow{2}{*}{ Chilgok } & $\mathrm{M}$ & Garden type & Appreciation & Fixed type & Marble, Glass \\
\hline & $\mathrm{W}$ & Garden type & Appreciation & Fixed type & Marble, Glass \\
\hline \multirow{2}{*}{ Tongdosa } & $\mathrm{M}$ & Garden type & Appreciation & Fixed type & Stone \\
\hline & W & - & - & - & - \\
\hline
\end{tabular}

${ }^{\mathrm{z}} \mathrm{M}=\mathrm{Men}$ 's restroom; $\mathrm{W}=$ Women's restsroom.

Area (Tongyeong direction) on Tongyeong-Daejeon Jungbu Expressway, men's restroom had the interior landscape formed with artificial flowers for appreciation in fixed form, using preservative wood. The others such as Insam Land and Hamyang Service Area were garden type installed for appreciation in fixed form, using marble (Table 11). The interior 
Table 5. Installation and usage type of indoor garden in Namhae expressway service areas.

\begin{tabular}{|c|c|c|c|c|c|}
\hline \multicolumn{2}{|c|}{ Name of service area } & \multirow{2}{*}{$\frac{\text { Installation type }}{\text { Garden type }}$} & \multirow{2}{*}{$\begin{array}{l}\text { Usage type } \\
\text { Appreciation }\end{array}$} & \multirow{2}{*}{$\frac{\text { Mobility }}{\text { Fixed type }}$} & \multirow{2}{*}{$\frac{\text { Material of planter }}{\text { Marble }}$} \\
\hline & $\mathrm{M}^{\mathrm{z}}$ & & & & \\
\hline Minulsall & W & Garden type & Appreciation & Fixed type & Marble \\
\hline \multirow{2}{*}{ Seomjingang } & M & Garden type & Appreciation & Fixed type & Marble, Preservative wood \\
\hline & W & - & - & - & - \\
\hline \multirow{2}{*}{ Jin-yeong } & $\mathrm{M}$ & Garden type & Appreciation & Fixed type & Glass \\
\hline & W & Garden type & Appreciation & Fixed type & Glass \\
\hline \multirow{2}{*}{ Haman } & $\mathrm{M}$ & Garden type & Appreciation & Fixed type & Glass \\
\hline & $\mathrm{W}$ & Garden type & Appreciation & Fixed type & Glass \\
\hline
\end{tabular}

${ }^{\mathrm{z}} \mathrm{M}=$ Men's restroom; $\mathrm{W}=$ Women's restroom.

Table 6. Installation and usage type of indoor garden in Donghean expressway service areas.

\begin{tabular}{|c|c|c|c|c|c|}
\hline \multicolumn{2}{|c|}{ Name of service area } & Installation type & Usage type & Mobility & Material of planter \\
\hline \multirow{2}{*}{ Gujeong } & $\mathrm{M}^{\mathrm{z}}$ & Garden type & Appreciation & Fixed type & Preservative wood, Glass \\
\hline & W & Garden type & Appreciation & Fixed type & Glass \\
\hline \multirow{2}{*}{ Donghae } & M & - & - & - & - \\
\hline & W & Garden type & Appreciation & Fixed type & Marble \\
\hline \multirow{2}{*}{ Okgye } & M & Garden type & Appreciation & Fixed type & Marble \\
\hline & W & Garden type & Appreciation & Fixed type & Marble \\
\hline
\end{tabular}

${ }^{\mathrm{z}} \mathrm{M}=$ Men's restroom; $\mathrm{W}=$ Women's restroom.

Table 7. Installation and usage type of indoor garden in Seohaean expressway serviece areas.

\begin{tabular}{|c|c|c|c|c|c|}
\hline \multicolumn{2}{|c|}{ Name of service area } & \multirow{2}{*}{$\frac{\text { Installation type }}{\text { Garden type }}$} & \multirow{2}{*}{$\begin{array}{c}\text { Usage type } \\
\text { Appreciation }\end{array}$} & \multirow{2}{*}{$\frac{\text { Mobility }}{\text { Fixed type }}$} & \multirow{2}{*}{$\frac{\text { Material of planter }}{\text { Marble }}$} \\
\hline & $\mathrm{M}^{\mathrm{z}}$ & & & & \\
\hline Goniาi & $\mathrm{W}$ & Garden type & Appreciation & Fixed type & Glass \\
\hline \multirow{2}{*}{ Daecheon } & M & Garden type & Appreciation & Fixed type & Marble \\
\hline & W & Garden type & Appreciation & Fixed type & Glass \\
\hline \multirow{2}{*}{ Seosan } & M & Garden type & Appreciation & Fixed type & Marble \\
\hline & W & Garden type & Appreciation & Fixed type & Marble, Brick \\
\hline \multirow{2}{*}{ Seocheon } & $\mathrm{M}$ & Garden type & Appreciation & Fixed type & Marble \\
\hline & W & Garden type & Appreciation & Fixed type & Marble \\
\hline \multirow{2}{*}{ Haengdamdo } & M & Parallel type & Appreciation & Fixed type & Preservative wood \\
\hline & W & Parallel type & Appreciation & Fixed type & Preservative wood \\
\hline \multirow{2}{*}{ Hongseong } & M & - & - & - & - \\
\hline & $\mathrm{W}$ & Garden type & Appreciation & Fixed type & Marble \\
\hline
\end{tabular}

${ }^{\mathrm{z}} \mathrm{M}=\mathrm{Men}$ 's restroom; W=Women's restroom. 
Table 8. Installation and usage type of indoor garden in Youngdong expressway service areas.

\begin{tabular}{|c|c|c|c|c|c|}
\hline \multicolumn{2}{|c|}{ Name of service area } & Installation type & Usage type & Mobility & Material of planter \\
\hline \multirow{2}{*}{ Gangneung } & $\mathrm{M}^{\mathrm{z}}$ & Garden type & Appreciation & Fixed type & Marble, Glass \\
\hline & W & Garden type & Appreciation & Fixed type & Marble \\
\hline \multirow{2}{*}{ Deokpyeong Ecoland } & $\mathrm{M}$ & Garden type & Appreciation & Fixed type & Glass \\
\hline & W & Garden type & Appreciation & Fixed type & Glass \\
\hline \multirow{2}{*}{ Munmak } & $\mathrm{M}$ & Garden type & Appreciation & Fixed type & Marble \\
\hline & W & Garden type & Appreciation & Fixed type & Marble \\
\hline \multirow{2}{*}{ Hoengseong } & M & Garden type & Appreciation & Fixed type & Marble \\
\hline & W & Garden type & Appreciation & Fixed type & Marble \\
\hline
\end{tabular}

${ }^{\mathrm{z}} \mathrm{M}=\mathrm{Men}$ 's restroom; $\mathrm{W}=$ Women's restroom.

Table 9. Installation and usage type of indoor garden in Jungbunaeryuk expressway service areas.

\begin{tabular}{|c|c|c|c|c|c|}
\hline \multicolumn{2}{|c|}{ Name of service area } & \multirow{2}{*}{$\frac{\text { Installation type }}{\text { Garden type }}$} & \multirow{2}{*}{$\begin{array}{l}\text { Usage type } \\
\text { Appreciation }\end{array}$} & \multirow{2}{*}{$\begin{array}{c}\text { Mobility } \\
\text { Fixed type }\end{array}$} & \multirow{2}{*}{$\frac{\text { Material of planter }}{\text { Marble }}$} \\
\hline Goesan & $\mathrm{M}^{\mathrm{z}}$ & & & & \\
\hline (Yangpyeong direction) & $\mathrm{W}$ & Garden type & Appreciation & Fixed type & Marble \\
\hline \multirow{2}{*}{ Namseongju } & $\mathrm{M}$ & Garden type & Appreciation & Fixed type & Marble, Glass \\
\hline & W & Garden type & Appreciation & Fixed type & Marble, Glass \\
\hline \multirow{2}{*}{ Mungyeong } & $\mathrm{M}$ & Garden type & Appreciation & Fixed type & Marble \\
\hline & $\mathrm{W}$ & Garden type & Appreciation & Fixed type & Marble \\
\hline \multirow{2}{*}{ Yeongsan } & M & Garden type & Appreciation & Fixed type & Marble \\
\hline & W & Garden type & Appreciation & Fixed type & Marble \\
\hline \multirow{2}{*}{ Chungju } & $\mathrm{M}$ & Garden type & Appreciation & Fixed type & Marble \\
\hline & $\mathrm{W}$ & Garden type & Appreciation & Fixed type & Marble \\
\hline \multirow{2}{*}{ Chilseo } & $\mathrm{M}$ & Aquarium type & Appreciation & Fixed type & Marble, Glass \\
\hline & $\mathrm{W}$ & Aquarium type & Appreciation & Fixed type & Marble, Glass \\
\hline \multirow{2}{*}{$\begin{array}{l}\text { Hyunpoong } \\
\text { (Daegu direction) }\end{array}$} & $\mathrm{M}$ & Garden type & Appreciation & Fixed type & Preservative wood \\
\hline & $\mathrm{W}$ & Garden type & Appreciation & Fixed type & Preservative wood \\
\hline \multirow{2}{*}{$\begin{array}{l}\text { Hyunpoong } \\
\text { (Hyunpoong direction) }\end{array}$} & $\mathrm{M}$ & Artificial flower type & Appreciation & Fixed type & Marble \\
\hline & $\mathrm{W}$ & Garden type & Appreciation & Fixed type & Glass \\
\hline
\end{tabular}

${ }^{\mathrm{z}} \mathrm{M}=$ Men's restroom; $\mathrm{W}=$ Women's restroom.

landscapes installed in men's and women's restrooms on Honam Expressway service areas were also garden type for appreciation in fixed form, mostly using glass and marble for planter materials (Table 12).

The results of this study showed that most interior landscapes installed in service areas are mostly garden type for simple appreciation, thereby not displaying their functions properly (Yun et al., 2016). Therefore, it is necessary to improve practicality in the resting places of service areas and implement various designs that are refreshing and sophisticated (Bang et al., 2013), and to develop types of interior landscapes in expressway service areas considering irrigation control where it is easy to maintain and replace indoor plants. 
Table 10. Installation and usage type of indoor garden in Jungang expressway service areas.

\begin{tabular}{|c|c|c|c|c|c|}
\hline \multicolumn{2}{|c|}{ Name of service area } & \multirow{2}{*}{$\frac{\text { Installation type }}{\text { Garden type }}$} & \multirow{2}{*}{$\begin{array}{l}\text { Usage type } \\
\text { Appreciation }\end{array}$} & \multirow{2}{*}{$\frac{\text { Mobility }}{\text { Fixed type }}$} & \multirow{2}{*}{$\frac{\text { Material of planter }}{\text { Glass }}$} \\
\hline Gunwi & $\mathrm{M}^{\mathrm{z}}$ & & & & \\
\hline (Busan direction) & W & Garden type & Appreciation & Fixed type & Marble, Glass \\
\hline \multirow{2}{*}{$\begin{array}{l}\text { Gunwi } \\
\text { (Chuncheon direction) }\end{array}$} & M & Garden type & Appreciation & Fixed type & Marble \\
\hline & W & Garden type & Appreciation & Fixed type & Marble, Glass \\
\hline \multirow{2}{*}{$\begin{array}{l}\text { Danyang } \\
\text { (Busan direction) }\end{array}$} & M & Garden type & Appreciation & Fixed type & Marble \\
\hline & W & - & - & - & - \\
\hline \multirow{2}{*}{$\begin{array}{l}\text { Dongmyeong } \\
\text { (Chuncheon direction) }\end{array}$} & M & Garden type & Appreciation & Fixed type & Marble \\
\hline & $\mathrm{W}$ & Garden type & Appreciation & Fixed type & Marble, Glass \\
\hline \multirow{2}{*}{$\begin{array}{l}\text { Andong } \\
\text { (Busan direction) }\end{array}$} & M & - & - & - & - \\
\hline & $\mathrm{W}$ & Planter-box type & Appreciation & Portable type & \\
\hline \multirow{2}{*}{$\begin{array}{l}\text { Andong } \\
\text { (Chuncheon direction) }\end{array}$} & M & Garden type & Appreciation & Fixed type & Marble \\
\hline & $\mathrm{W}$ & Garden type & Appreciation & Fixed type & Marble \\
\hline \multirow{2}{*}{$\begin{array}{l}\text { Chiak } \\
\text { (Busan direction) }\end{array}$} & M & - & - & - & - \\
\hline & W & Garden type & Appreciation & Fixed type & Marble \\
\hline \multirow{2}{*}{$\begin{array}{l}\text { Chiak } \\
\text { (Chuncheon direction) }\end{array}$} & M & - & - & - & - \\
\hline & W & Garden type & Appreciation & Fixed type & Marble \\
\hline
\end{tabular}

${ }^{\mathrm{z}} \mathrm{M}=$ Men's restroom; W=Women's restroom.

Table 11. Installation and usage type of indoor garden in Tongyeong-daejeon Jungbu expressway service areas.

\begin{tabular}{|c|c|c|c|c|c|}
\hline \multicolumn{2}{|c|}{ Name of service area } & Installation type & Usage type & Mobility & Material of planter \\
\hline \multirow{2}{*}{$\begin{array}{l}\text { Deogyusan } \\
\text { (Tongyeong direction) }\end{array}$} & $\mathrm{M}^{\mathrm{z}}$ & Artificial flower type & Appreciation & Fixed type & Preservative wood \\
\hline & W & - & - & - & - \\
\hline \multirow{2}{*}{$\begin{array}{l}\text { Insam Land } \\
\text { (Tongyeong direction) }\end{array}$} & M & Garden type & Appreciation & Fixed type & Marble \\
\hline & W & Garden type & Appreciation & Fixed type & Marble \\
\hline \multirow{2}{*}{$\begin{array}{l}\text { Hamyang } \\
\text { (Tongyeong direction) }\end{array}$} & M & Garden type & Appreciation & Fixed type & Marble \\
\hline & W & Garden type & Appreciation & Fixed type & Marble \\
\hline
\end{tabular}

${ }^{\mathrm{z}} \mathrm{M}=\mathrm{Men}$ 's restroom; $\mathrm{W}=$ Women's restroom.

Table 12. Installation and usage type of indoor garden in Honam expressway service areas.

\begin{tabular}{|c|c|c|c|c|c|}
\hline \multicolumn{2}{|c|}{ Name of service area } & \multirow{2}{*}{$\begin{array}{c}\text { Installation type } \\
\text { Garden type }\end{array}$} & \multirow{2}{*}{$\begin{array}{c}\text { Usage type } \\
\text { Appreciation }\end{array}$} & \multirow{2}{*}{$\begin{array}{c}\text { Mobility } \\
\text { Fixed type }\end{array}$} & \multirow{2}{*}{$\frac{\text { Material of planter }}{\text { Marble }}$} \\
\hline & $\mathrm{M}^{\mathrm{z}}$ & & & & \\
\hline Gokseong & W & Garden type & Appreciation & Fixed type & Marble \\
\hline \multirow{2}{*}{ Juam } & M & Garden type & Appreciation & Fixed type & Marble \\
\hline & $\mathrm{W}$ & Garden type & Appreciation & Fixed type & Marble \\
\hline
\end{tabular}

${ }^{\mathrm{z}} \mathrm{M}=$ Men's restroom; W=Women's restroom. 
Table 13. Soil conditions and introduced material for indoor garden in Namhae expressway service areas.

\begin{tabular}{|c|c|c|c|}
\hline \multicolumn{2}{|c|}{ Name of service area } & Garden ornament & Soil \\
\hline \multirow{2}{*}{ Munsan } & $\mathrm{M}^{\mathrm{z}}$ & - & Field soil \\
\hline & $\mathrm{W}$ & - & Field soil \\
\hline \multirow{2}{*}{ Seomjingang } & M & Cylindrical wooden fences, Thatched house model, Doll model & Field soil \\
\hline & W & - & Field soil \\
\hline \multirow{2}{*}{ Jin-yeong } & $\mathrm{M}$ & - & Field soil \\
\hline & W & - & Field soil \\
\hline \multirow{2}{*}{ Haman } & M & - & Field soil, white gravel \\
\hline & W & - & Field soil, white gravel \\
\hline
\end{tabular}

${ }^{\mathrm{z}} \mathrm{M}=\mathrm{Men}$ 's restroom; $\mathrm{W}=$ Women's restroom.

Table 14. Soil conditions and introduced material for indoor garden in Donghaean expressway service areas.

\begin{tabular}{|c|c|c|c|}
\hline \multicolumn{2}{|c|}{ Name of service area } & Garden ornament & Soil \\
\hline \multirow{2}{*}{ Gujeong } & $\mathrm{M}^{\mathrm{z}}$ & \multirow{6}{*}{ - } & Field soil \\
\hline & W & & Field soil \\
\hline \multirow{2}{*}{ Donghae } & M & & Field soil \\
\hline & W & & Field soil \\
\hline \multirow{2}{*}{ Okgye } & M & & Field soil \\
\hline & W & & Field soil \\
\hline
\end{tabular}

${ }^{\mathrm{z}} \mathrm{M}=\mathrm{Men}$ 's restroom; W=Women's restroom.

Table 15. Soil conditions and introduced material for indoor garden in Seohaean expressway service areas.

\begin{tabular}{|c|c|c|c|}
\hline \multicolumn{2}{|c|}{ Name of service area } & Garden ornament & Soil \\
\hline \multirow[b]{2}{*}{ Goindol } & $\mathrm{M}^{\mathrm{z}}$ & Pond & Field soil \\
\hline & $\mathrm{W}$ & $\begin{array}{c}\text { Cage } \\
\text { Stepping Bridge stone } \\
\text { Stone pagoda }\end{array}$ & Field soil \\
\hline \multirow{2}{*}{ Daecheon } & M & \multirow{2}{*}{-} & Field soil \\
\hline & $\mathrm{W}$ & & Field soil \\
\hline \multirow[t]{2}{*}{ Seosan } & M & \multirow[t]{2}{*}{-} & $\begin{array}{l}\text { White gravel } \\
\text { Black gravel }\end{array}$ \\
\hline & W & & Field soil \\
\hline \multirow{2}{*}{ Seocheon } & M & \multirow{2}{*}{-} & Field soil \\
\hline & W & & Field soil \\
\hline \multirow{2}{*}{ Haengdamdo } & M & \multirow{2}{*}{-} & Field soil \\
\hline & W & & Field soil \\
\hline \multirow{2}{*}{ Hongseong } & M & - & Field soil \\
\hline & W & & Field soil \\
\hline
\end{tabular}

${ }^{\mathrm{z}} \mathrm{M}=$ Men's restroom; $\mathrm{W}=$ Women's restroom. 


\section{Interior landscape garden ornaments and soil in expressway service areas}

Types of garden ornaments for some interior landscapes installed in expressway service areas of each route include wooden fences, stones, pond, Korean traditional totem pole symbolizing love and hope, and ginseng model, and also other materials such as light, bird cage, stone pagoda, male and female figures, duck model, and water mill. Most common soil type was field soil, decorated with white or black gravel on top (Table 13-20). Recently, service areas in some regions succeeded in increasing the rate of use and customer satisfaction by repairing the facilities, creating a space with a distinctive

Table 16. Soil conditions and introduced material for indoor garden in Youngdong expressway service areas.

\begin{tabular}{|c|c|c|c|}
\hline \multicolumn{2}{|c|}{ Name of service area } & Garden ornament & Soil \\
\hline \multirow{2}{*}{ Gangneung } & $\mathrm{M}^{\mathrm{z}}$ & - & Field soil \\
\hline & W & - & Field soil \\
\hline \multirow{2}{*}{ Deokpyeong Ecoland } & M & & Field soil \\
\hline & W & - & Field soil \\
\hline \multirow{2}{*}{ Munmak } & M & Light & Field soil \\
\hline & W & - & Field soil \\
\hline \multirow{2}{*}{ Hoengseong } & M & $\begin{array}{c}\text { Stone pagoda, Duck model, Wooden } \\
\text { Fences }\end{array}$ & Field soil \\
\hline & W & Stone pagoda & $\begin{array}{l}\text { White gravel } \\
\text { Field soil }\end{array}$ \\
\hline
\end{tabular}

${ }^{\mathrm{z}} \mathrm{M}=\mathrm{Men}$ 's restroom; $\mathrm{W}=$ Women's restroom.

Table 17. Soil conditions and introduced material for indoor garden in Jungbunaeryuk expressway service areas.

\begin{tabular}{|c|c|c|c|}
\hline Name of se & & Garden ornament & Soil \\
\hline \multirow{2}{*}{$\begin{array}{l}\text { Goesan } \\
\text { (Yangpyeong direction) }\end{array}$} & $\mathrm{M}^{\mathrm{z}}$ & - & $\begin{array}{l}\text { Color gravel } \\
\text { White gravel }\end{array}$ \\
\hline & W & - & Field soil \\
\hline \multirow{2}{*}{ Namseongju } & $\mathrm{M}$ & - & White gravel \\
\hline & W & - & White gravel \\
\hline \multirow{2}{*}{ Mungyeong } & M & - & Field soil \\
\hline & W & - & Field soil \\
\hline \multirow{2}{*}{ Yeongsan } & $\mathrm{M}$ & - & Field soil \\
\hline & W & - & Field soil \\
\hline \multirow{2}{*}{ Chungju } & M & - & Field soil \\
\hline & W & - & Field soil \\
\hline \multirow{2}{*}{ Chilseo } & $\mathrm{M}$ & - & Field soil \\
\hline & W & - & Field soil \\
\hline \multirow{2}{*}{$\begin{array}{l}\text { Hyunpoong } \\
\text { (Daegu direction) }\end{array}$} & M & Light & White gravel \\
\hline & W & Light & Gravel \\
\hline
\end{tabular}

${ }^{\mathrm{z}} \mathrm{M}=$ Men's restroom; $\mathrm{W}=$ Women's restroom. 
Table 18. Soil conditions and introduced material for indoor garden in Jungang expressway service areas.

\begin{tabular}{|c|c|c|c|}
\hline \multicolumn{2}{|c|}{ Name of service area } & Garden ornament & Soil \\
\hline \multirow{2}{*}{ Gunwi } & $\mathrm{M}^{\mathrm{z}}$ & Wooden Fences & Field soil \\
\hline & W & - & Field soil \\
\hline \multirow{2}{*}{ Danyang } & M & Stone & Field soil \\
\hline & W & Stone & Field soil \\
\hline \multirow{2}{*}{ Dongmyeong } & M & - & Field soil \\
\hline & W & Stone & Field soil \\
\hline \multirow{2}{*}{ Andong } & M & $\begin{array}{c}\text { Millstone } \\
\text { Stone pagoda }\end{array}$ & $\begin{array}{l}\text { Color gravel } \\
\text { White gravel }\end{array}$ \\
\hline & W & $\begin{array}{c}\text { Toad model } \\
\text { Stone }\end{array}$ & Field soil \\
\hline \multirow{2}{*}{ Chiak } & M & - & Field soil \\
\hline & W & Stone water mill & Field soil \\
\hline
\end{tabular}

${ }^{\mathrm{z}} \mathrm{M}=\mathrm{Men}$ 's restroom; $\mathrm{W}=$ Women's restroom.

Table 19. Soil conditions and introduced material for indoor garden in Tongyeong-daejeon Jungbu expressway service areas.

\begin{tabular}{|c|c|c|c|}
\hline \multicolumn{2}{|c|}{ Name of service area } & Garden ornament & Soil \\
\hline \multirow{2}{*}{$\begin{array}{l}\text { Deogyusan } \\
\text { (Tongyeong direction) }\end{array}$} & $\mathrm{M}^{\mathrm{z}}$ & - & Field soil \\
\hline & $\mathrm{W}$ & - & Field soil \\
\hline \multirow{2}{*}{ Insam Land } & M & - & Field soil \\
\hline & $\mathrm{W}$ & Ginseng model & Field soil \\
\hline \multirow{2}{*}{ Hamyang } & M & Thatched house model Water mill & Field soil \\
\hline & $\mathrm{W}$ & - & Field soil \\
\hline
\end{tabular}

${ }^{\mathrm{z}} \mathrm{M}=$ Men's restroom; $\mathrm{W}=$ Women's restroom.

Table 20. Soil conditions and introduced material for indoor garden in Honam expressway service areas.

\begin{tabular}{|c|c|c|c|}
\hline \multicolumn{2}{|c|}{ Name of service area } & Garden ornament & Soil \\
\hline \multirow{2}{*}{ Gokseong } & $\mathrm{M}^{\mathrm{z}}$ & - & Field soil \\
\hline & W & light & Field soil \\
\hline \multirow{2}{*}{ Juam } & M & $\begin{array}{l}\text { Duck model } 2 \\
\text { Korean traditional totem poles symbolizing symbol of love and hope }\end{array}$ & Field soil \\
\hline & $\mathrm{W}$ & $\begin{array}{c}\text { Korean traditional totem pole } 2 \\
\text { Birds model } 5\end{array}$ & Field soil \\
\hline
\end{tabular}

${ }^{\mathrm{z}} \mathrm{M}=\mathrm{Men}$ 's restroom; W=Women's restroom.

theme of regional characteristics and using regional tourism resources by making good use of the eco-friendly architecture and surrounding natural environment, thereby improving their competitive edge compared to other service areas (Lee and Kim, 2011). The results of the study also showed that Deokpyeong, Insam Land, Goindol and Seomjingang Service Areas had interior landscapes well installed in restrooms using various items that represent the regional attributes (Fig. 1). 


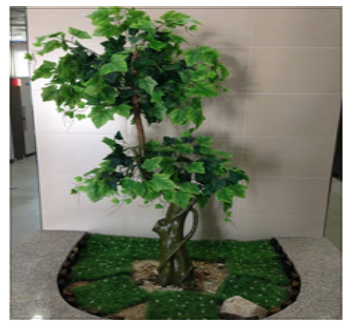

Geoncheon

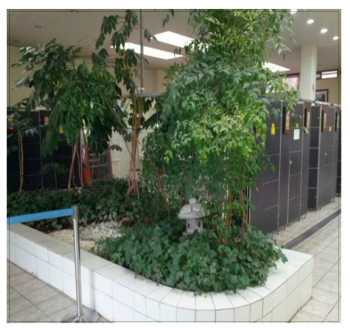

Anseong

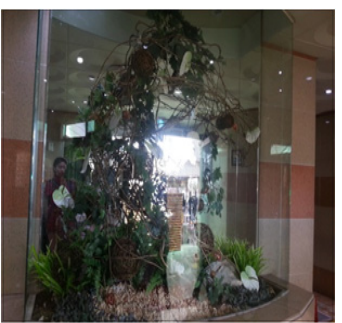

Chilgok

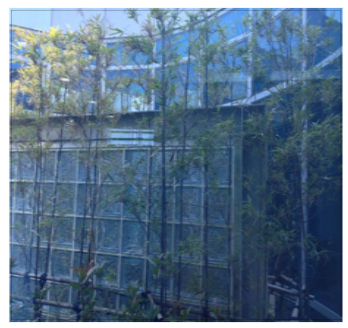

Jin-yeong

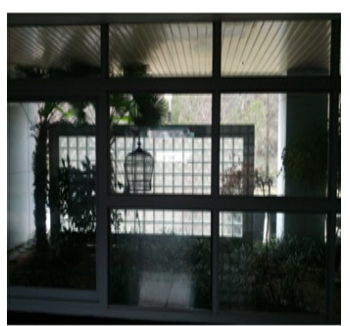

Goindol

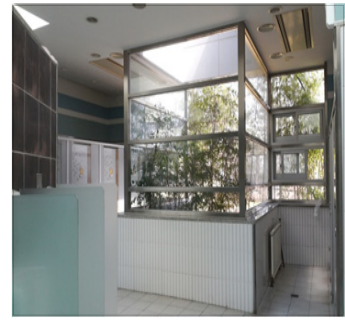

Gimcheon

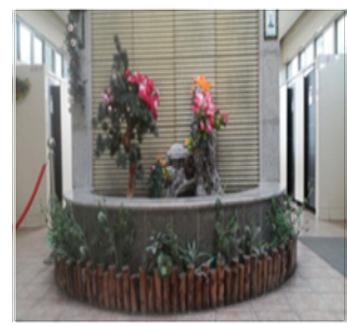

Okcheon

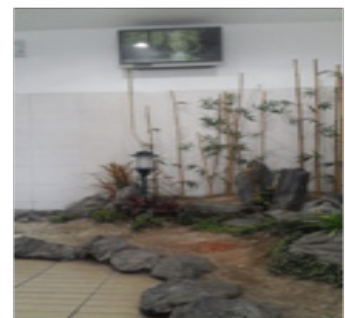

Tongdosa

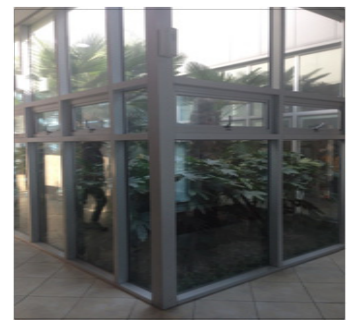

Haman

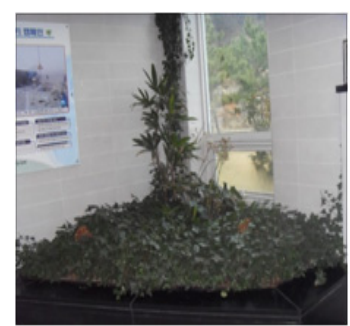

Daecheon

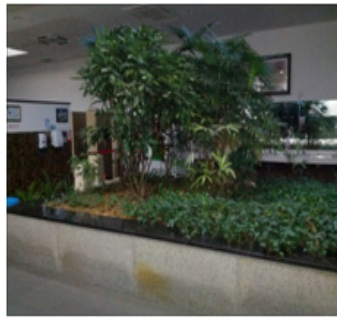

Manghyang

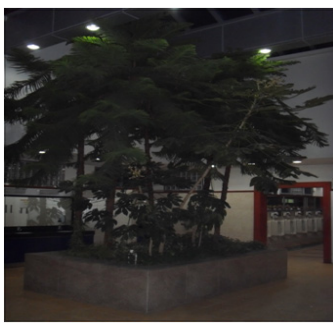

Jugam

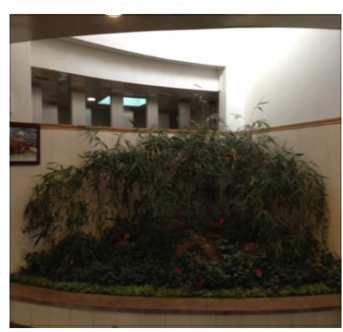

Munsan

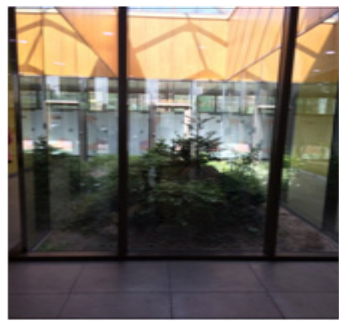

Gujeong

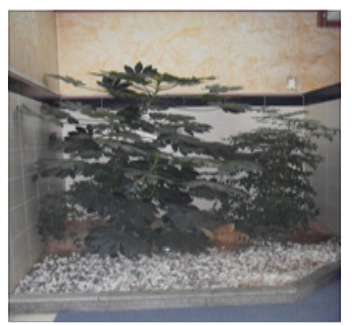

Seosan

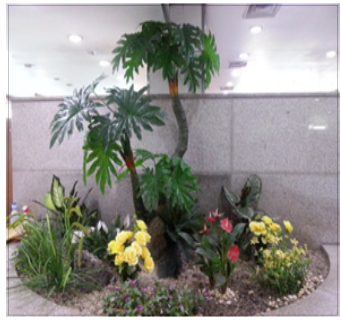

Sintanjin

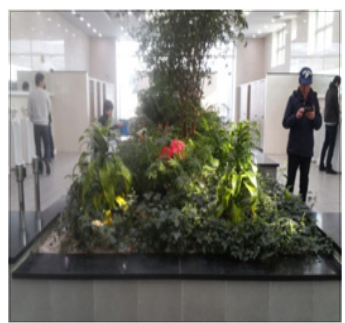

Cheongju

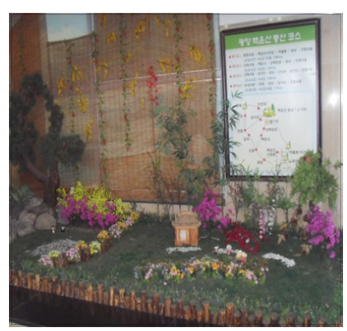

Seomjingang

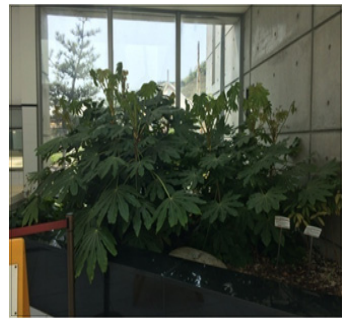

Okgye

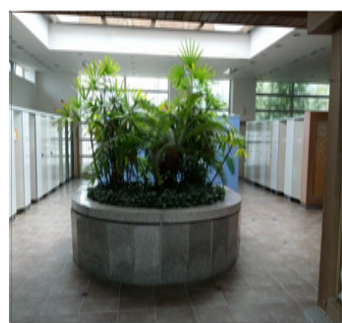

Seocheon

Figure 1. Pictures of different indoor gardens in expressway service areas.

As for the soil, there are studies on light artificial soil combinations with excellent ability to remove contaminants from the air that are also good for plant growth (Jang et al., 2013). Accordingly, it seems necessary to replace interior landscape soil in service areas that is mostly field soil with the kind that has functionality to improve the environment and is also economically feasible. 


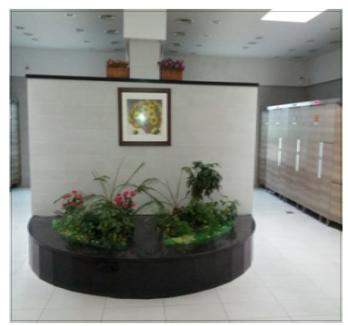

Hongseong

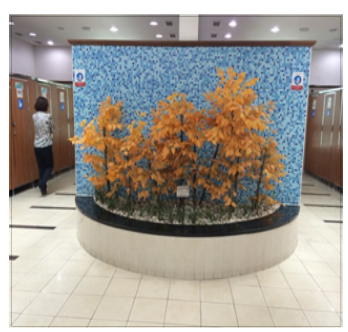

Goesan (Yangpyeong)

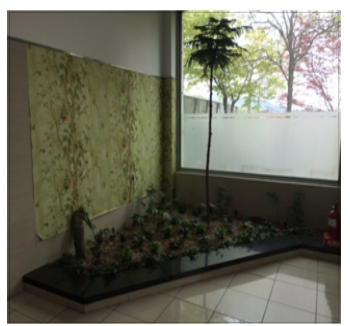

Chungju

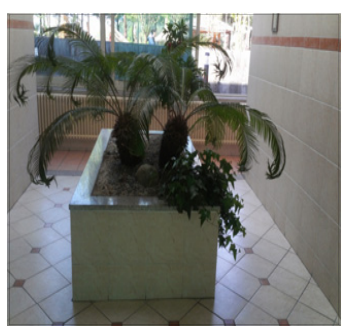

Danyang

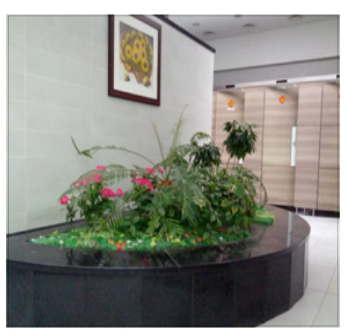

Hamyang

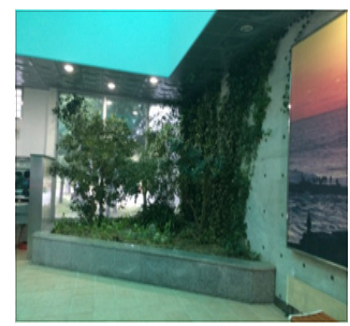

Gangneung

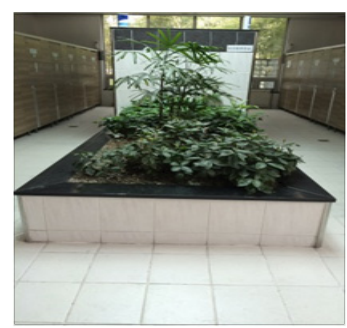

Namseongju

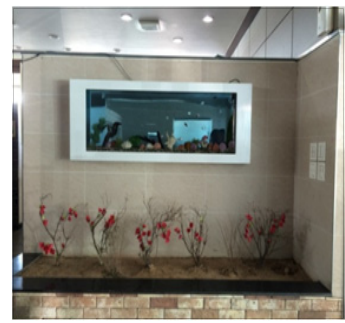

Chilseo

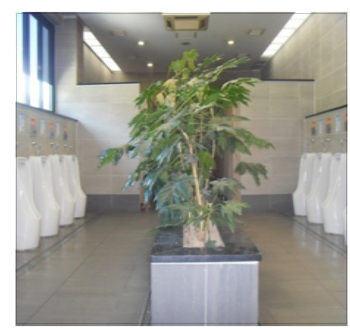

Dongmyeong

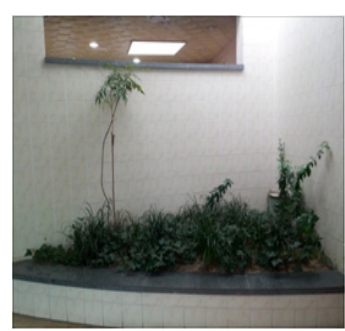

Gokseong

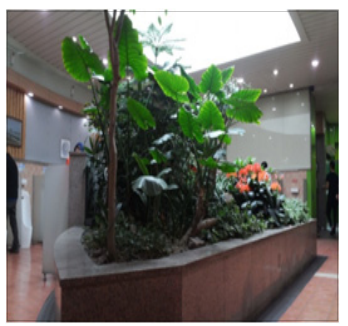

Munmak

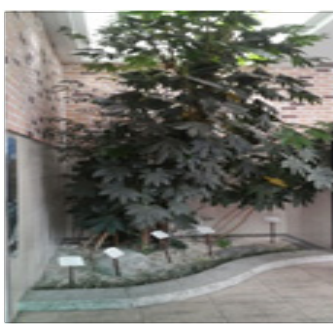

Mungyeong

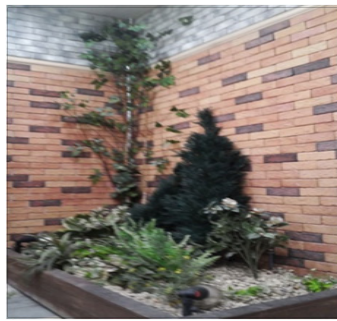

Hyunpoong

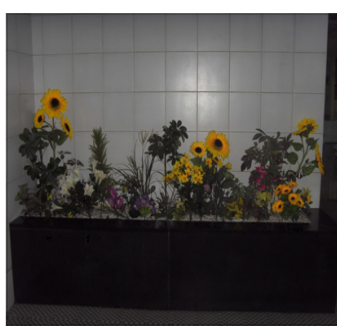

Deogyusan (Tongyeong)

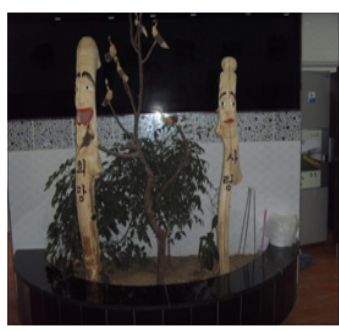

Juam (Cheonan)

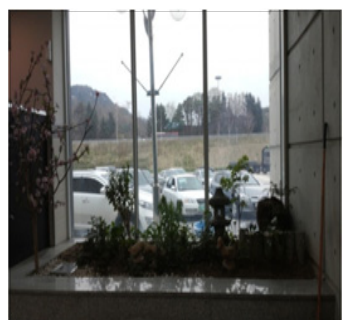

Hoengseong

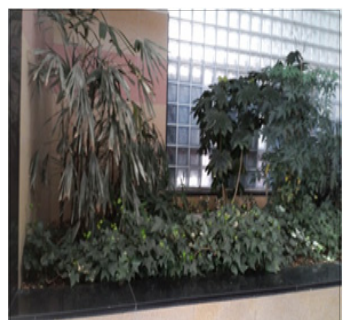

Yeongsan

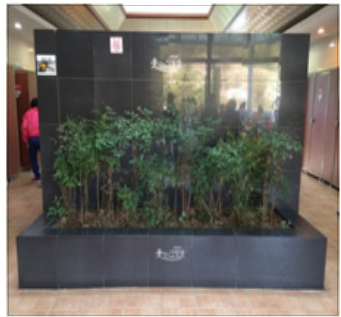

Gunwi

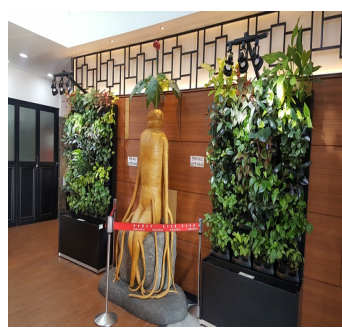

Insam Land

Figure 1. Continued.

\section{Cultivated plants in expressway service areas and photographs of interior landscape}

There were total 30 species, 27 genus and 19 families planted in interior landscapes of expressway service areas (Table 21). They were classified into upper, middle, lower and flowering plants according to tree height and planting type (Yoon, 1989; Lee, 1996). 
Table 21. Introduced plants of indoor garden in expressway service areas.

\begin{tabular}{|c|c|}
\hline Planting Type & Name of Plant \\
\hline Upper tree & $\begin{array}{c}\text { Chamaedorea elegans, Cycas revoluta, Fatsia japonica, Phyllostachys bambusoides, Phyllostachys nigra, } \\
\text { Pseudosasa japonica, Rhapis excelsa, Rhapis humilis, Ternstroemia gymnanthera (Wight et Arn.) Sprague, } \\
\text { Washingtonia filifera (L. Linden) H. Wendl. }\end{array}$ \\
\hline Middle tree & $\begin{array}{l}\text { Araucaria heterophylla, Ardisia crenata Sims, Ardisia japonica (Thunb.) Blume., Aucuba japonica, Forsythia } \\
\text { koreana, Nandina domestica Thunb., Ophiopogon japonicus, Rhododendron schlippenbachii, Schefflera arboricola }\end{array}$ \\
\hline Lower tree & $\begin{array}{c}\text { Euphorbia pulcherrima, Farfugium japonicum, Hedera helix, Hedera rhombea, Hosta plantaginea (Lam.) Asche, } \\
\text { Liriope platyphylla, Pachysandra terminalis, Pteridium aquilinum var. latiusculum }\end{array}$ \\
\hline Flowering plant & Aquilegia buergeriana var. oxysepala, Kerria japonica for. plena, Viola tricolor var. hortensis \\
\hline
\end{tabular}

There were 10 species, 9 genus and 6 families of upper plants, such as Chamaedorea elegans, Cycas revoluta, Fatsia japonica, Phyllostachys bambusoides, Phyllostachys nigra, Pseudosasa japonica, Rhapis excelsa, Rhapis humilis, Ternstroemia gymnanthera (Wight et Arn.) Sprague, and Washingtonia filifera (L. Linden) H. Wendl. For middle plants, there were 9 species, 8 genus and 8 families such as Araucaria heterophylla, Ardisia crenata Sims, Ardisia japonica (Thunb.) Blume., Aucuba japonica, Forsythia koreana, Nandina domestica Thunb., Ophiopogon japonicus, Rhododendron schlippenbachii, and Schefflera arboricola. There were 8 species, 7 genus and 6 families for lower plants such as Euphorbia pulcherrima, Farfugium japonicum, Hedera helix, Hedera rhombea, Hosta plantaginea (Lam.) Asche, Liriope platyphylla, Pachysandra terminalis, and Pteridium aquilinum var. latiusculum. As for flowering plants, there were 3 species, 3 genus and 3 families such as Aquilegia buergeriana var. oxysepala, Kerria japonica for. plena, and Viola tricolor var. hortensis.

A study by Jung et al. (2006) on the current state of interior landscape plants in 28 expressway service areas in Korea showed that there were 44 species and 26 families, but this study presented that there were 10 species and 7 families fewer. This may be because some plants withered away due to the poor environmental conditions at the entrance or lack of light in the restrooms, or also because they were replaced with plants that are more convenient to maintain.

\section{Conclusion}

This study determined the current state of interior landscaping by visiting expressway service areas of 147 service areas on 189 expressways in Korea. There were 56 service areas with interior landscapes, showing a ratio of 38\%. On Gyeongbu Expressway with the biggest number of service areas, 16 (47\%) out of 34 service areas had interior landscapes installed. Other routes had 10 or fewer. Moreover, 35 expressway service areas were decorated with natural flowers, 7 with artificial flowers, and 14 with a combination of both natural and artificial flowers. Expressway interior landscapes were mostly installed in the restrooms: 47 in men's restrooms and 50 in women's restrooms. The most common type of interior landscape was fixed garden type, and 3 of them had portable planters installed. Most interior landscapes did not have resting facilities installed around them, with focus mostly on interior landscape itself to simply watch and appreciate it.

There were total 30 species, 27 genus and 19 families planted in interior landscapes of expressway service areas. It turned out that there were 10 species and 7 families fewer than previous research. This indicates that it is necessary to implement various plants that present seasonality and beauty of plant colors while also well enduring the indoor environment.

Recently, there is more and more resting space as expressway service areas seek various changes in terms of function. However, there are very few interior landscapes in service areas compared to exterior landscapes. It is necessary to install 
the interior landscapes, which are limited only to men's and women's restrooms, to other spaces such as souvenir shop, cafeteria and information center. Furthermore, there is a need for various interior landscape designs that display the beauty of plants as well as artistic functions and environmental control beyond just garden type. It is also desirable to come up with a distinctive interior landscape to lead to specialized regional service areas. Visitors can enjoy both a spectacle as well as natural landscape if resting places are installed around the interior landscape, thereby improving the image of service areas, helping visitors relieve fatigue of both body and soul, and providing a pleasant environment.

\section{References}

Bang, S.W., J.E. Song, Y.E. Park, J.Y. Kim, and K.J. Kim. 2013. Evaluation of design preference on the functional Plant Pot. J. Korean Soc. People Plants Environ. 16(6):485-491. DOI:10.11628/ksppe.2013.16.6.485

Choi, H.G. and Y.H. Lee. 2014. The impact of service quality and management abilities on customer satisfaction, customer loyalty, sales performance in expressway service area business. Acad. Customer Satisf. Manag. 16(2):79-104.

Jang, T.J., H.Y. Kim, and K.B Lim. 2013. Selection of artificial media suitable for the growth of foliage plants for indoor vertical garden. Flower Res. J. 21(1):11-16. DOI:10.11623/frj.2013.21.1.1

Jung, J., M.H. Kwon, and K.J. Bang. 2006 A study on the maintenance condition of the interior landscaping plants in the restroom of the expressway service area: focus on the main expressway in Korea. J. Korean Soc. People Plants Environ. 9(4):140-147.

Korea Expressway Corporation. 2017. 2017 Service Area. Retrieved from http://www.ex.co.kr/site/com/pageProcess.do

Kwon, Y.I., C.W. Lee, J.K. Lim, and B.D. Jung. 2010. A study on user's characteristics and selection factors of the highway service area. J. Transp. Res. 17(4):75-84.

Lee, W.M., B.H. Han, J.Y. Kim, and S.C. Park. 2015. A study of the functional improvement for comfortable expressway service areas : metropolitan expressway service areas. J. Korean Inst. Landsc. Archit. 43(3):63-76. DOI:10.9715/KILA. 2015.43.3.063

Lee, Y. 1996. The Colored Illustrated Book of the Korean Flora (pp.210-348). Seoul, Korea: Kyohaksa.

Lee, Y.N. and S.W. Kim. 2011. A study of improving the spatial design components of the green-space in the Yongdong expressway service station. J. Korean Soc. Des. Culture 17(1):466-481. DOI:10.18208/ksdc.2011.17.1.038

Shin, H.C., J.S. Yun, and K.O. Choi. 2013. Landscape formation states and the preference analyses of indoor garden in the working space. J. Recreat. Landsc. 7(2):43-52.

Yoon, P. 1989. The illustrated book of the Korean horticultural flora (pp.89-536). Paju, Korea: Jisik-Sanupsa.

Yun, J.S., J.S. Hwang, S.W. Ha, and K.O. Choi. 2016. The current status of indoor gardens creation in elementary, middle, and high-schools in Gyeongsangnam-do. J. East Asian Landsc. Stud. 10(2):37-47. 